\title{
Analyzing Weight Distribution of Feedforward Neural Networks and Efficient Weight Initialization*
}

\author{
Jinwook Go, Byungjoon Baek, and Chulhee Lee \\ Department of Electrical and Electronic Engineering, BERC, Yonsei University \\ 134 Shinchon-Dong, Seodaemoon-Gu, Seoul, Korea \\ Tel: (82-2) 2123-2779, Fax: (82-2) 312-4584 \\ chulhee@yonsei.ac.kr
}

\begin{abstract}
In this paper, we investigate and analyze the weight distribution of feedforward two-layer neural networks in order to understand and improve the time-consuming training process of neural networks. Generally, it takes a long time to train neural networks. However, when a new problem is presented, neural networks have to be trained again without any benefit from previous training. In order to address this problem, we view training process as finding a solution weight point in a weight space and analyze the distribution of solution weight points in the weight space. Then, we propose a weight initialization method that uses the information on the distribution of the solution weight points. Experimental results show that the proposed weight initialization method provides a better performance than the conventional method that uses a random generator in terms of convergence speed.
\end{abstract}

\section{Introduction}

Neural networks have been successfully applied in various fields such as pattern recognition, signal processing, and dynamic modeling. The increasing popularity of neural networks is partly due to their ability to learn and generalize. In particular, neural networks make no prior assumptions about the statistics of input data and can construct complex decision boundaries. The decision boundaries of a neural network are determined by a set of weights and the set of weights can be arranged as a vector, which can be represented as a weight point in the weight space that is defined by the weights. From this perspective, training process of neural networks may be viewed as finding a weight point that is optimal for a given problem (Fig. 1). We call such a weight point a solution weight point for a given problem. In practice, the solution weight point may not be unique. In other words, a weight point in the vicinity of the solution weight point may provide an equivalent performance and such weight points may form a region, which will be called a solution region in this paper (Fig. 1).

This work was supported in part by Biometrics Engineering Research Center (BERC) at Yonsei University (KOSEF). 
If the neural network is trained with a different initial weight point, the resulting weight point can be quite different, though the outputs of the neural network are similar. The behavior of neural networks that produce similar outputs with very different sets of weights leads us to suspect that there may exist several solution regions for a given problem. From this perspective, we explore the possibility to speed up the training process of neural networks by utilizing the information on the distribution of solution regions in the weight space, assuming that such information is available. In other words, by analyzing and accumulating the information on the distribution of solution regions in weight space for various problems, we may initialize neural networks more effectively for a new problem.

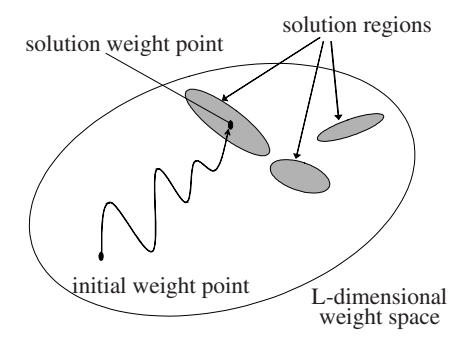

Fig. 1. Solution weight point and solution regions in the weight space.

Efficient weight initialization is one of the most important factors for fast convergence and generalization, and many authors have proposed various weight initialization methods. The simplest and most widely used weight initialization method is a random initialization assuming some probability distributions and some researchers proposed several modified methods to determine the best initialization interval [1-2]. Another initialization approach is to incorporate the known prior knowledge into weight initialization [3]. Generally, it has been shown that the weight initialization is a factor that may influence generalization results. Also, an analysis of the weight distribution may provide a way to reduce the complexity of neural networks [4]. However, the weight distribution in a trained neural network is not well understood, which is due to a strong problem dependency for the weight distribution [5]. In this paper, we analyze the distribution of solution regions for two-pattern class classification problems. From this analysis, we present a new insight into the weight distribution and propose a new weight initialization method that uses the information on the weight distribution.

\section{Feedforward Neural Networks and Terminologies}

We will briefly discuss the structure of feedforward neural networks that will be used in the experiments. A typical neural network has an input layer, a number of hidden layers, and an output layer. It may also include bias terms. Fig. 2 shows an example of two-layer feedforward neural networks with two outputs. In Fig. 2, let 
$X_{\text {in }}=\left(x_{1}, x_{2}, \cdots, x_{M}\right)^{T}$ be the input vector and $Y=\left(y_{1}, y_{2}\right)^{T}$ the output vector. The bias vector is given by $B=\left(b_{1}, b_{2}\right)^{T}$. Without loss of generality, we can set $b_{1}=b_{2}=1$. Then we may include the bias term in the input layer as follows:

$$
X=\left(x_{1}, x_{2}, \cdots, x_{M}, 1\right)^{T}=\left(x_{1}, x_{2}, \cdots, x_{M}, x_{M+1}\right)^{T} \text { where } x_{M+1}=1
$$

If we assume that there are $K$ neurons in the hidden layer, the weight matrices $W_{1}$ and $W_{2}$ for the two-pattern class neural network can be represented by

$$
W_{1}=\left[\begin{array}{cccc}
w_{1,1}^{h i} & w_{1,2}^{h i} & \ldots & w_{1, M+1}^{h i} \\
w_{2,1}^{h i} & w_{2,2}^{h i} & \ldots & w_{2, M+1}^{h i} \\
: & : & \ldots & : \\
w_{K, 1}^{h i} & w_{K, 2}^{h i} & \ldots & w_{K, M+1}^{h i}
\end{array}\right] \quad W_{2}=\left[\begin{array}{llll}
w_{1,1}^{o h} & w_{1,2}^{o h} & \ldots & w_{1, K+1}^{o h} \\
w_{2,1}^{o h} & w_{2,2}^{o h} & \ldots & w_{2, K+1}^{o h}
\end{array}\right]
$$

where $w_{j, i}^{h i}$ is the weight between input neuron $i$ and hidden neuron $j$ and $w_{k, j}^{o h}$ is the weight between hidden neuron $j$ and output neuron $k$. All the elements of $W_{1}$ and $W_{2}$ can be arranged as a weight vector $W$. In other words,

$$
W=\left(w_{1,1}^{h i}, w_{1,2}^{h i}, \ldots, w_{K, M+1}^{h i}, w_{1,1}^{o h}, w_{1,2}^{o h}, \ldots, w_{2, K+1}^{o h}\right)^{T}=\left(w_{1}, w_{2}, w_{3}, \ldots, w_{L}\right)^{T}
$$

where $L=((M+1) K+2(K+1))$. Then, we may view the weight vector $W$ as a weight point in an $L$-dimensional weight space that is defined by all the weights in the neural network. From this perspective, training a neural network may be viewed as finding a weight point that produces a desirable sequence of output vectors for a given sequence of input vectors. In this paper, such a weight point will be called a solution weight point. The solution weight point is located within the solution region, where the set of solution weight points is distributed densely in the weight space [6].

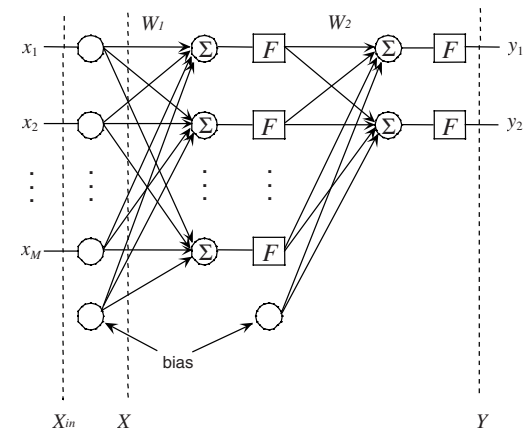

Fig. 2. An example of two-layer feedforward neural networks (two-pattern classes).

\section{Weight Distribution in the Weight Space}

Even for a simple neural network, the number of weights can be large. In order to make the computation manageable, we chose a neural network that has 2 input neu- 
rons, 3 hidden neurons, 2 output neurons, and 2 bias terms. The total number of weights of the neural network is 17 and the training process is equivalent to finding a solution weight point in a 17-dimensional weight space. The backpropagation algorithm is used for training and the decision rule is to select the class corresponding to the output neuron with the largest output [7].

In the following experiment, tests are conducted using generated data whose statistics are estimated from real remotely-sensed data collected from the field spectrometer system (FSS) assuming Gaussian distribution [8]. The 50 pairs of two classes were selected randomly from Table 1 and feature selection is applied to the original 60dimensional data, producing a 2-dimensional input vector. Each input is preprocessed so that its mean value is zero and the inputs are scaled so that they are in the range of -2 to 2 . For each two-pattern classification problem, the network was trained with 1000 different initial weight points until the difference between the classification error and the Bayes' error is smaller than 2\%, resulting in 1000 solution weight points. The Bayes' error is estimated using the Gaussian ML classifier. In this paper, we will call the set of solution weight points for each problem a solution set.

Table 1. Description of the multi-temporal 15 classes.

\begin{tabular}{cccc}
\hline \hline Class \# & Date & Species & No. of samples \\
\hline \hline 1 & 770508 & Winter Wheat & 691 \\
2 & 780515 & Spring Wheat & 474 \\
3 & 771026 & Winter Wheat & 393 \\
4 & 771018 & Spring Wheat & 313 \\
5 & 780921 & Winter Wheat & 292 \\
6 & 780816 & Native Grass Pas & 212 \\
7 & 780726 & Summer Fallow & 200 \\
8 & 780709 & Summer Fallow & 190 \\
9 & 780921 & Oats & 182 \\
10 & 780816 & Oats & 165 \\
11 & 771018 & Oats & 161 \\
12 & 770626 & Grain Sorghum & 157 \\
13 & 780515 & Summer Fallow & 150 \\
14 & 770920 & Spring Wheat & 129 \\
15 & 780602 & Barley & 112 \\
\hline
\end{tabular}

\subsection{Eigenvalues and Determinant of Covariance Matrix of the Solution Set}

In order to analyze the distribution of the solution sets, we investigate the eigenvalues and determinant of the covariance matrix estimated from the solution sets. Table 2 shows the largest ten eigenvalues (ordered by size) of the covariance matrix estimated from the 50 solution sets (1,000 solution weight points per set, a total of 50,000 solution weight points), as well as the proportions and accumulations of the eigenvalues. As can be seen in Table 2, the largest six eigenvalues account for more than $90 \%$ of the total energy. In particular, the largest three eigenvalues are dominant and the rest are small in value. It appears that most variations of solution weight points in weight space occur along a few eigenvectors corresponding to the largest eigenvalues and there is little variation along the other eigenvectors. This indicates that, assuming a normal distribution, the data will be distributed in the shape of an elongated hyperel- 
lipsoid with its center at the mean of the solution sets. The principal axes of this hyperellipsoid are given by the eigenvectors of the covariance matrix of the solution sets and the energies contained in these axes are proportional to the corresponding eigenvalues. Fig. 3(a) shows the distribution of the 50 solution sets projected onto the 3 dimensional space spanned by the three eigenvectors corresponding to the largest eigenvalues of the covariance matrix. It can be observed that solution weight points seem to form a single cluster with a dense spot in the center and are distributed closely around the center of the cluster. In addition, as there are little differences among the largest three eigenvalues in Table 2, it seems that the solution weight points are distributed in the shape of a sphere instead of an ellipsoid in the 3 dimensional space spanned by the three eigenvectors corresponding to the largest eigenvalues. Fig. 3(b) shows the histogram of the individual weight values of all the 50 solution sets and the histogram is compared with a normal distribution. The mean and the variance are 0.105 and 25.2, respectively. From Fig. 3(b), the weight values do not seem to be normally distributed, though quite often they are assumed to have a normal distribution [5].

Table 2. Eigenvalues of covariance matrix of 50 solution sets.

\begin{tabular}{|l|r|r|r|r|r|r|r|r|r|r|}
\hline & 1 & 2 & 3 & 4 & 5 & 6 & 7 & 8 & 9 & 10 \\
\hline Eigenvalue & 114.8 & 113.8 & 108.2 & 17.4 & 17.1 & 16.2 & 12.3 & 12.3 & 11.6 & 2.9 \\
\hline Proportion (\%) & 26.9 & 26.7 & 25.3 & 4.0 & 4.0 & 3.8 & 2.9 & 2.9 & 2.7 & 0.7 \\
\hline Accumulation (\%) & 26.9 & 53.6 & 78.9 & 82.9 & 86.9 & 90.7 & 93.6 & 96.5 & 99.2 & 99.9 \\
\hline
\end{tabular}

(a)

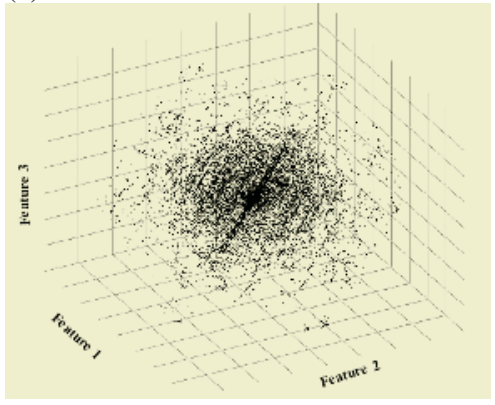

(b)

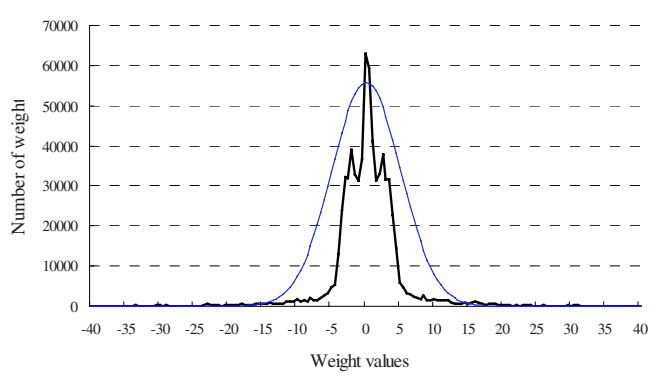

Fig. 3. (a) Three dimensional projection map of the 50 solution sets. (b) histogram of weights and the corresponding normal distribution for the 50 solution sets.

Fig. 4 shows the determinants of the covariance matrices for each of the 50 solution sets. The determinant of a covariance matrix is equal to the product of all eigenvalues and the determinant of a covariance matrix provides the information about the actual volume where the data are distributed. From Fig. 4, it can be said that there are significant differences among the solution sets, indicating that there are significant differences in the actual volumes where the solution sets are distributed. 


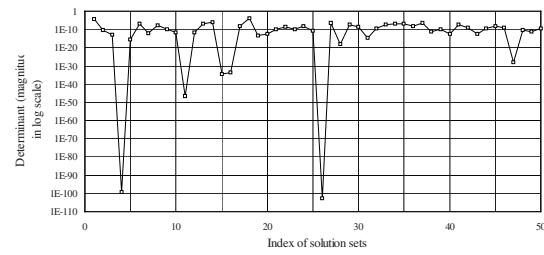

Fig. 4. Determinants of the 50 solution sets.

\subsection{Distribution of Solution Region in the Weight Space}

A solution set (the set of solution weight points for each problem) is not homogeneous in the sense that the weight points inside the volume containing the solution set do not provide the same classification accuracy. Thus, we need to find homogeneous subsets of a solution set in order to analyze the distribution of solution regions. In order to find homogeneous subsets inside a solution set, we applied clustering to each solution set using the Euclidean distance. It is noted that by rearranging the hidden neurons and corresponding weights, we can produce 6 different configurations of neural networks that produce the exactly same output. This indicates that there exist 6 equivalent weight points in the 17 dimensional weight space for the neural network (2 input neurons, 3 hidden neurons, 2 output neurons, and 2 bias terms). The number of equivalent weight points corresponds to the factorial of the number of hidden neurons. In the analysis of the weight distribution, these equivalent weight points should be treated as the same point, though their locations are different in the weight space. In order to assign equivalent weight points to the same point, we compared the 3 weight values between the bias term in the input layer and the hidden neurons, and rearranged the hidden neurons and corresponding weights such that $w_{1} \geq w_{2} \geq w_{3}$, where $w_{i}$ is the weight value between the bias term in the input layer and the $i$-th hidden neuron. After this rearrangement of the hidden neurons and corresponding weights, clustering was applied. The resulting subsets are homogeneous in the sense that if we choose any weight point from the volume that contains a subset, the point provides the maximum classification accuracy without training. In this paper, we will call the homogeneous subset a homogeneous solution region. Clustering results show that there exist a number of homogeneous solution regions in a solution set and also there is a large variation among the numbers of homogeneous solution regions of the solution sets.

Since clustering was applied to just 1000 solution weight points, the resulting clusters may not be an accurate estimation of the distribution of solution regions. In the next step, we expand each homogeneous solution region to find the maximum volume such that any weight point within the volume provides the maximum classification accuracy. In other words, by expanding a homogeneous solution region, we try to find the maximum volume that contains the homogeneous solution region.

We propose the following expansion procedure for expanding a homogeneous solution region. First, we try to expand a homogeneous solution region along the eigen- 
vector corresponding to the smallest eigenvalue of the covariance matrix estimated from a homogeneous solution region. After doubling the smallest eigenvalue, 1000 weight points are generated assuming the Gaussian distribution for the homogeneous solution region with the increased eigenvalue. Then, we compute the classification accuracy for the generated weight points. If the number of the generated weight points whose classification accuracy is within $2 \%$ of the maximum classification accuracy is greater than 995 , which corresponds to $99.5 \%$ of the total generated points, we determine that the expansion is valid and the modified eigenvalue is retained. Otherwise, the original eigenvalue is retained. The same procedure is repeated for the rest of the eigenvalues in increasing order. Finally, 1000 weight points are generated with all the updated eigenvalues and only weight points whose classification accuracy is within $2 \%$ of the maximum classification accuracy are retained. From the new weight points, the mean vector and covariance matrix are estimated. This whole procedure is repeated until the eigenvalues cannot be increased any more.

After the expansion procedure, the volume of a homogeneous solution region increases substantially. Table 3 shows the largest seven eigenvalues of the homogeneous solution region after the expansion procedure. In this case, the volume of the homogeneous solution region increased about $10^{124}$ times. And most solution weight points are distributed in a few major components, indicating that there are high correlations between adjacent weight values. Table 3 also shows corresponding angles between the eigenvectors before expansion and the corresponding eigenvectors after expansion. The large angles indicate that the shape of the homogeneous solution region also changed substantially.

Table 3. Eigenvalues of the homogeneous solution region and angles between eigenvectors after expansion procedure.

\begin{tabular}{|l|r|r|r|r|r|r|}
\hline & 1 & 2 & 3 & 4 & 5 & 6 \\
\hline Eigenvalue & 36.7 & 11.6 & 3.0 & 0.8 & 0.3 & 0.1 \\
\hline Proportion (\%) & 69.8 & 22.2 & 5.6 & 1.5 & 0.7 & 0.2 \\
\hline Accumulation (\%) & 69.8 & 92.0 & 97.6 & 99.1 & 99.8 & 99.9 \\
\hline Angles between eigenvectors (degree) & 8 & 74 & 104 & 78 & 77 & 43 \\
\hline
\end{tabular}

\section{Weight Initialization Using the Distribution of Solution Regions}

Now we explore the possibility to initialize feedforward neural networks with prior knowledge that was obtained by investigating the distribution of solution regions in the weight space. In other words, we wish to use the information on the weight distribution to provide a good initial weight point.

In the previous analysis, we obtained 2581 homogeneous solution regions for the 50 pattern classification problems. We computed the mean vector and the covariance matrix of each homogeneous solution region. From these statistics, we generated 300 weight points for each homogeneous solution region assuming the Gaussian distribution. Thus, the total number of solution weight points is 774,300 , to which we applied 
K-means clustering in order to divide the solution weight points into 100 clusters whose mean vectors may be used as initial weight points for a new problem.

In order to examine whether this information of weight distribution is useful for selecting a good initial weight point, we initialized the neural network with the cluster means for 780 new classification problems that were not used to obtain the 100 clusters. The new classification problems consist of 780 pairs of two classes from the combination of 40 classes. Fig. 5(a) shows the distribution of the 40 classes and Fig. 5(b) shows the distribution of the 15 classes shown in Table 1.

(a)

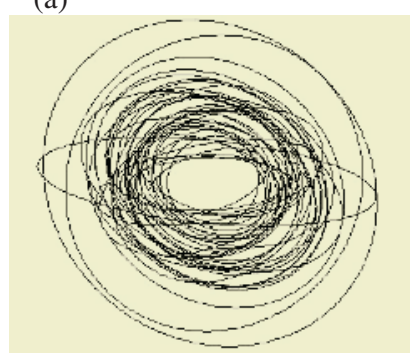

(b)

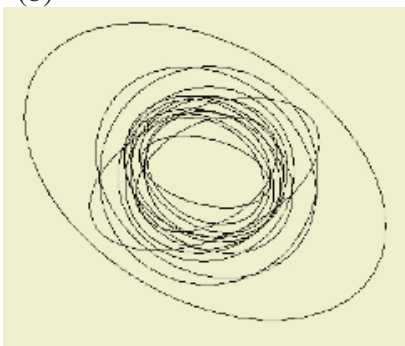

Fig. 5. (a) Distribution of 40 classes used for the 780 two-class problems, (b) distribution of 15 classes described in Table 1.

The mean vectors of the 100 clusters were used as initial weight points and then we selected the weight point that provides the best classification accuracy. The classification accuracy was estimated using 25 randomly selected samples from 500 training samples of each class, which correspond to $5 \%$ of the total training samples. Thus, the extra computation time is relatively minor. Random initialization assuming a uniform distribution over the interval from -0.5 to 0.5 was also tested to compare the performance of the proposed initialization method.

Table 4 shows the differences between the classification accuracies that were obtained using the mean vectors of the clusters as initial weights and the classification accuracies that were obtained using random initialization for the 780 new problems. The classification accuracies were obtained without any training. In $82.2 \%$ of the 780 problems, the proposed initialization method achieves higher classification accuracy than the random initialization method. It appears that the solution regions retain some useful information to solve new problems.

Fig. 6 shows the average classification accuracies of the 780 problems. As can be seen, the proposed method significantly outperforms the random weight initialization. After one iteration, the average classification accuracy obtained using the mean vectors of the clusters as initial weights was $81.58 \%$ for training data. On the other hand, the average classification accuracy obtained using randomly selected initial weights was $71.82 \%$. The experiments show that the knowledge on the solution distribution can provide helpful information for a new problem. However, for a few problems, using the mean vectors of the clusters as initial weights was ineffective. 
Table 4. The classification accuracy differences between the initial weights selected from cluster means and the random initial weights without any training.

\begin{tabular}{|c|r|r|r|}
\hline \hline Difference (D) & No. of problems & Proportion (\%) & Accumulation (\%) \\
\hline \hline $\mathrm{D} \geq 30 \%$ & 24 & 3.08 & 3.08 \\
\hline $30 \%>\mathrm{D} \geq 20 \%$ & 103 & 13.21 & 16.28 \\
\hline $20 \%>\mathrm{D} \geq 10 \%$ & 227 & 29.10 & 45.38 \\
\hline $10 \%>\mathrm{D}>0 \%$ & 311 & 39.87 & 82.18 \\
\hline $0 \%>\mathrm{D} \geq-5 \%$ & 92 & 11.79 & 97.05 \\
\hline$-5 \%>\mathrm{D} \geq-10 \%$ & 20 & 2.56 & 99.62 \\
\hline$-10 \%>\mathrm{D} \geq-15 \%$ & 3 & 0.38 & 100.00 \\
\hline
\end{tabular}

(a)

(b)
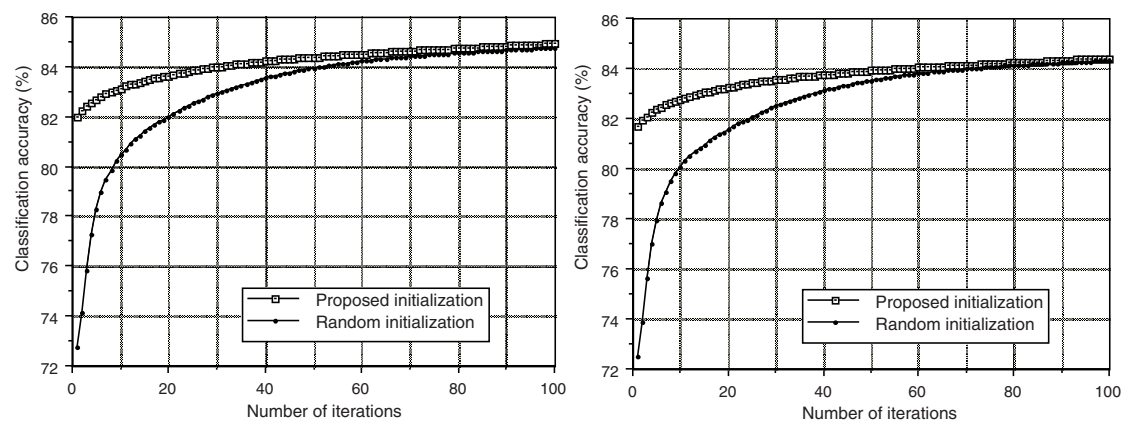

Fig. 6. Performance comparison between the proposed initialization using the information on the solution distribution and the random initialization (averages of the 780 experiments). (a) training data, (b) test data.

\section{Conclusion}

In this paper, by analyzing weight distribution of neural networks, we explored the possibility to speed up training process. First, we obtained 50,000 solution weight points for 50 two-pattern-class classification problems and found a number of homogeneous solution regions in the weight space by applying clustering to the solution weight points. Then, the homogeneous solution regions were expanded in order to obtain the maximum volume for each homogeneous solution region. By analyzing distribution of the homogeneous solution regions, we found that most weights are distributed along a few major directions, indicating that there are very high correlations between weight values of the homogeneous solution regions. Finally, we explored the possibility to use this prior information on the distribution of weights for efficient weight initialization. Experimental results indicate that the weight analysis may provide useful information on selecting initial weights. As the knowledge on the weight distribution accumulates, it would be possible to select good initial weights for a new problem. 


\section{References}

1. L. F. A. Wessels and E. Barnard: Avoiding false local minima by proper initialization of connections. IEEE Trans. Neural Networks, Vol. 3. (1992) 899-905

2. G. P. Drago and S. Ridella: Statistically controlled activation weight initialization (SCAWI). IEEE Trans. Neural Networks, Vol. 3. (1992) 627-631

3. Z. Chen, T. Feng, and Z. Houkes: Incorporating a priori knowledge into initialized weights for neural classifier. Proc. Int. Joint Conf. Neural Networks, Vol. 2. (2000) 291-296

4. S. J. Nowlan and G. E. Hinton: Simplifying neural networks by soft weight-sharing. Neural Computation, Vol. 4, No. 4. (1992) 473-493

5. I. Bellido and E. Fiesler: Do backpropagation trained neural networks have normal weight distributions? Proc. Int. Conf. Artificial Neural Networks. (1993) 772-775

6. J. A. Richards: Remote Sensing Digital Image Analysis. Springer-Verlag. (1993)

7. R. P. Lippmann: An introduction to computing with neural nets. IEEE ASSP Magazine, Vol. 4. (1987) 4-22

8. L. L. Biehl and et. al.: A crops and soils data base for scene radiation research. Proc. Machine Process. of Remotely Sensed Data Symp., West Lafayette, Indiana. (1982) 169-177 